\title{
Visible Near-infrared Spectral Evolution of Irradiated Mixed Ices and Application to Kuiper Belt Objects and Jupiter Trojans
}

\author{
Michael J. Poston ${ }^{1,2,3}$ (D), Ahmed Mahjoub ${ }^{1,2}$ (D), Bethany L. Ehlmann ${ }^{1,2}$ (iD) Jordana Blacksberg $^{2}$, Michael E. Brown ${ }^{1}$, \\ Robert W. Carlson ${ }^{2}$, John M. Eiler ${ }^{1}$, Kevin P. Hand ${ }^{2}$ (D), Robert Hodyss ${ }^{2}$, and Ian Wong ${ }^{1}$ (i) \\ ${ }_{1}^{1}$ California Institute of Technology, Pasadena, CA, USA; michael.poston@swri.org \\ 2 Jet Propulsion Laboratory, California Institute of Technology, Pasadena, CA, USA \\ Received 2017 September 8; revised 2018 January 11; accepted 2018 February 14; published 2018 March 30
}

\begin{abstract}
Understanding the history of Kuiper Belt Objects and Jupiter Trojans will help to constrain models of solar system formation and dynamical evolution. Laboratory simulations of a possible thermal and irradiation history of these bodies were conducted on ice mixtures while monitoring their spectral properties. These simulations tested the hypothesis that the presence or absence of sulfur explains the two distinct visible near-infrared spectral groups observed in each population and that Trojans and KBOs share a common formation location. Mixed ices consisting of water, methanol, and ammonia, in mixtures both with and without hydrogen sulfide, were deposited and irradiated with $10 \mathrm{keV}$ electrons. Deposition and initial irradiation were performed at $50 \mathrm{~K}$ to simulate formation at 20 au in the early solar system, then heated to Trojan-like temperatures and irradiated further. Finally, irradiation was concluded and resulting samples were observed during heating to room temperature. Results indicated that the presence of sulfur resulted in steeper spectral slopes. Heating through the 140-200 K range decreased the slopes and total reflectance for both mixtures. In addition, absorption features at 410,620, and $900 \mathrm{~nm}$ appeared under irradiation, but only in the $\mathrm{H}_{2} \mathrm{~S}$-containing mixture. These features were lost with heating once irradiation was concluded. While the results reported here are consistent with the hypothesis, additional work is needed to address uncertainties and to simulate conditions not included in the present work.
\end{abstract}

Key words: astrochemistry - Kuiper Belt: general - minor planets, asteroids: individual (Jupiter Trojans) planets and satellites: surfaces - radiation: dynamics

\section{Introduction}

One of the three "cross-cutting themes" of the Vision and Voyages Planetary Science Decadal Survey for 2013-2022 is the understanding of our solar system's beginnings. What were the initial conditions of the solar system? Was there large-scale migration of the giant planets and related disruption of small bodies? Small bodies (comets, asteroids, Trojans, and Kuiper Belt Objects (KBOs)) are perhaps the best resources available to answer these fundamental questions.

Jupiter Trojans are a large resonant population of asteroids situated at the L4 and L5 Lagrange points along Jupiter's orbit around the Sun. Present information about Trojan surfaces comes primarily from full-disk photometry and spectroscopy. Two key observables are (1) the albedo and (2) the slope of the spectra of reflected sunlight in the visible and near-infrared (VNIR) ranges of the spectrum, often called the "color" of the asteroid. Several groups have measured the visible and/or nearinfrared color of the Trojans and found a bimodal distribution consistent with two dynamically well-mixed subpopulations (Roig et al. 2008; Emery et al. 2011; Wong et al. 2014). Here these subpopulations will be called R (red) and LR (less red), signifying the relative magnitude of the positive spectral slope observed over the VNIR range. Significantly, Centaurs and Kuiper Belt Objects (KBOs) of similar size to Trojans also appear to have a bimodal distribution of visible colors (Brown et al. 2011; Wong \& Brown 2016, 2017), albeit with somewhat different color groups, referred to here as VR (very red) and R (red). The Trojans overall have lower albedos than KBOs, and the LR and R Trojan subpopulations have comparable albedos.

\footnotetext{
${ }^{3}$ Now located at Southwest Research Institute, San Antonio, TX.
}

In contrast, the VR KBOs have higher albedos than the R KBOs.

Brown et al. (2011) hypothesized that the color bimodality of the Centaurs and small KBOs is due to differences in surface composition. In their model, a volatile ice species that condensed on an object's surface is stable against thermal loss for some characteristic time when beyond its "sublimation line." This same species is thermally unstable at smaller orbital radii and will not remain at the surface (outermost meters) long enough to form detectable irradiation products. Therefore, subpopulations inside or outside the sublimation line that initially accreted with identical compositions would have formed refractory irradiation crusts incorporating different reagents, and thus, different products. These two subpopulations were then mixed by dynamical instability (such as was described in Morbidelli et al. 2005 and Nesvorný et al. 2013) to create the present-day populations.

Wong \& Brown (2016) modeled sublimation lines in the early solar system and found that the hydrogen sulfide $\left(\mathrm{H}_{2} \mathrm{~S}\right)$ sublimation line was within the primordial planetesimal disk, at a temperature of approximately $50 \mathrm{~K}$ and a distance of approximately $20 \mathrm{au}$. Thus, they put forth the hypothesis that $\mathrm{H}_{2} \mathrm{~S}$ was the key species involved in forming the redder Trojan/KBO spectral groups. In this hypothesis, objects formed on either side of the $\mathrm{H}_{2} \mathrm{~S}$ sublimation line and built up their VR or R irradiation crusts at those locations. After the irradiation crusts had formed, dynamical instability scattered some of the objects to other locations in the solar system, such as the locations of the present-day Trojans and KBOs. Wong \& Brown (2016) hypothesized that movement closer to the Sun warmed the objects and converted them to R and LR. 
A necessary aspect of the color biomodality hypothesis is large-scale reorganization of the solar system; therefore, support for the hypothesis would also support the hypothesis of past large-scale reorganization of the solar system (but says nothing about the converse). If this occurred, then small bodies that formed in the outer solar system may be found in the inner solar system (e.g., Levison et al. 2009). For example, dwarf planet Ceres may have originated in the outer solar system and moved in during a dynamical instability (e.g., McKinnon 2008; McCord et al. 2011; DeSanctis et al. 2015). Should the largescale reorganization hypothesis prove true, it is likely that some small bodies in the inner solar system will have residual sulfuror nitrogen-containing compounds at their surfaces, as a tracer of this history.

Numerous laboratory simulations have been conducted examining the chemical behavior of pure and mixed ices under simulated space temperature, pressure, and irradiation conditions (e.g., Hudson et al. 2008; Bennett et al. 2013 and references therein). However, few studies have considered the spectral slope in the VNIR. Andronico et al. (1987) and Thompson et al. (1987) both examined the color of hydrocarbon residues in the visible, but not at temperatures and pressures appropriate to small bodies in the outer solar system. Brunetto et al. (2006) irradiated pure ices of methanol, methane, and benzene with ions of hundreds of kiloelectron volts of energy and examined their VNIR spectra in vacuum at cryogenic temperatures. Each hydrocarbon reddened at a different rate and with a different shape to its spectrum. Lebofsky \& Fegley (1976) irradiated pure films and mixtures of $\mathrm{H}_{2} \mathrm{~S}, \mathrm{NH}_{3}$, and $\mathrm{H}_{2} \mathrm{O}$ with $\mathrm{UV}$ photons in vacuum at cryogenic temperatures. They saw little change in spectral slope unless $\mathrm{H}_{2} \mathrm{~S}$ was included in the film. When $\mathrm{H}_{2} \mathrm{~S}$ was included, they observed an increase in slope shortward of $750 \mathrm{~nm}$ and a decrease in slope longward of $750 \mathrm{~nm}$, as well as the generation of absorption features in the VNIR wavelength range.

VNIR spectra of Jupiter's moon Io collected by the Solid State Imaging System (SSI) on the Galileo mission have been categorized into red, yellow, green, dark, and white color groups by Geissler et al. (1999). The red, yellow, dark, and white categories all have positive spectral slopes, inferred to result from sulfurous compounds. Europa, another nearby moon of Jupiter, has red patches on its trailing hemisphere, which likely contain sulfur compounds (Carlson et al. 2009). The chemistry of sulfur is complex, ranging from short chains, to rings, to massive polymers (e.g., Eckert \& Steudel 2003; Carlson et al. 2007, and references therein), but a common theme among most of the studied compounds is the appearance of red spectral slopes.

A necessary step to testing the hypothesis (that the inclusion of sulfur in the irradiation crust of the redder group of Trojans/ KBOs is the key difference between the subpopulations) is obtaining spectra of irradiated, relevant ice mixtures at relevant temperatures. While it has been previously shown that carboncontaining and sulfur-containing ices exhibit red spectral slopes upon irradiation, intimate mixtures of water, carbon, nitrogen, and sulfur compounds have not been examined under relevant conditions. The temperatures of interest in this work are $50 \mathrm{~K}$, where $\mathrm{H}_{2} \mathrm{~S}$ at the surfaces of recently formed bodies would be stable against sublimation for tens of millions of years, and 120 to $200 \mathrm{~K}$, reasonable temperatures for a Trojan asteroid to have experienced while moving closer to the Sun. Additionally, spectral slopes can change upon large radiation exposures (e.g.,
Andronico et al. 1987), which have not previously been studied at relevant conditions (the work of Lebofsky and Fegley delivered a small dose; total energy delivered was equivalent to less than a year at $5 \mathrm{au}$ under present solar wind irradiation). In order to simulate processes that would have occurred over billions of years, larger total doses are needed. The experiments reported here were conducted under conditions of composition, temperature, pressure, and radiation dose that were intended to simulate the hypothesized history of Trojan asteroids originating in the outer solar system.

\section{Equipment and Methods}

Laboratory simulations were carried out in the Minos vacuum chamber located in the Icy Worlds Simulation Laboratory at the Jet Propulsion Laboratory. The chamber has been described elsewhere (Hand 2007; Hand \& Carlson 2011, 2012). Briefly, the chamber was equipped with a closed-cycle helium cryostat, a Midac FTIR, an SRS residual gas analyzer, a Kimble Physics Electron Source, and a Princeton Instruments Acton grating spectrometer (with tungsten-halogen source). This paper focuses on results from the visible near-infrared spectrometer. The spectrometer had two gratings and two detectors, with a maximum range from about $350-1650 \mathrm{~nm}$, and employed a $16 \mathrm{~nm}$ data point spacing. VNIR spectra were collected on the visible detector in an automated fashion at $20 \pm 10$ minute increments throughout each experiment, and manually on the near-infrared detector as often as was practical. The spot size of the spectrometer was smaller than the area irradiated by the electrons. Typical chamber pressure after pumping overnight (the chamber was not baked) was $3 \times 10^{-8}$ torr, dropping to as low as $5 \times 10^{-9}$ torr with the cryostat in operation. Photons from the spectrometer sources entered the chamber above the horizontally mounted sample surface and were reflected toward the sample with a $22^{\circ}$ incidence and collection angle from the normal to the substrate surface.

Experiments were conducted in two optical geometries: specular and non-specular. The specular-geometry experiments were conducted in both "thin" and "thick" film experiments. Due to the scattering nature of the sample film, the specular geometry resulted in a mixed specular/diffusely scattered signal reaching the collection optics. A complication of the mixed specular/diffuse experimental geometry is the inability to distinguish changes in the scattering properties of the film from changes in absorbance. The only way to truly isolate the absorbance would be with an integrating sphere. However, performing the experiment in a complementary (non-specular) geometry provided insight into which changes resulted from changes in scattering versus changes in absorption. The nonspecular geometry involved switching the detectors from the specular orientation to a diffuse orientation.

Mixed ices of water $\left(\mathrm{H}_{2} \mathrm{O}\right)$, methanol $\left(\mathrm{CH}_{3} \mathrm{OH}\right)$, and ammonia $\left(\mathrm{NH}_{3}\right)$-with and without hydrogen sulfide $\left(\mathrm{H}_{2} \mathrm{~S}\right)$ - were deposited at $50 \mathrm{~K}$ on a pristine gold mirror target (hereafter " 3 -ice" and " 3 -ice $+\mathrm{H}_{2} \mathrm{~S}$ "). These ices have been observed in comets and encompass the major elemental constituents of icy primitive body surfaces. Deposition in the "thin, specular" experiments was to constant thickness, while in the "thick, specular" experiments it was continued until the signal exiting the chamber was approximately constant. The mixed ices for the "non-specular" experiments were deposited until a suitably strong signal arrived at the spectrometer, and the same signal strength was used for both films. In addition, 
Table 1

Key Parameters of Each Experiment

\begin{tabular}{|c|c|c|c|c|c|c|c|}
\hline \multicolumn{2}{|c|}{ Gas Mixture Composition } & \multicolumn{2}{|c|}{ Thickness $(\mu \mathrm{m})$} & \multicolumn{2}{|c|}{ Irradiation (hr) } & \multicolumn{2}{|c|}{ Total Dose $(\mathrm{eV} / 16 \mathrm{amu})$} \\
\hline Gases & Ratio & & $+/-$ & at $50 \mathrm{~K}$ & at $120 \mathrm{~K}$ & at $50 \mathrm{~K}$ & at $120 \mathrm{~K}$ \\
\hline $\mathrm{CH}_{3} \mathrm{OH}$ & pure & 2 & 1 & 20 & $1^{\mathrm{a}}$ & 600 & $30^{\mathrm{a}}$ \\
\hline \multicolumn{8}{|l|}{ Round 1: Specular } \\
\hline $\mathrm{H}_{2} \mathrm{O}: \mathrm{CH}_{3} \mathrm{OH}: \mathrm{NH}_{3}$ & $1: 2: 2$ & 2 & 1 & 20 & 1 & 600 & 30 \\
\hline $\mathrm{H}_{2} \mathrm{O}: \mathrm{CH}_{3} \mathrm{OH}: \mathrm{NH}_{3}: \mathrm{H}_{2} \mathrm{~S}$ & $1: 3: 3: 3$ & 2 & 1 & 20 & 1 & 600 & 30 \\
\hline \multicolumn{8}{|l|}{ Round 2: Specular } \\
\hline$\overline{\mathrm{H}_{2} \mathrm{O}: \mathrm{CH}_{3} \mathrm{OH}: \mathrm{N}_{3}}$ & $1: 2: 2$ & 40 & 10 & 24 & 29 & 700 & 900 \\
\hline $\mathrm{H}_{2} \mathrm{O}: \mathrm{CH}_{3} \mathrm{OH}: \mathrm{NH}_{3}: \mathrm{H}_{2} \mathrm{~S}$ & $1: 3: 3: 3$ & 200 & 80 & 170 & 260 & 5000 & 8000 \\
\hline \multicolumn{8}{|l|}{ Round 3: Non-specular } \\
\hline $\mathrm{H}_{2} \mathrm{O}: \mathrm{CH}_{3} \mathrm{OH}: \mathrm{NH}_{3}$ & $1: 2: 2$ & 8 & 3 & 71 & $73^{\mathrm{b}}$ & 2000 & $2000^{\mathrm{b}}$ \\
\hline $\mathrm{H}_{2} \mathrm{O}: \mathrm{CH}_{3} \mathrm{OH}: \mathrm{NH}_{3}: \mathrm{H}_{2} \mathrm{~S}$ & $1: 3: 3: 3$ & 60 & 20 & 77 & $69^{\mathrm{b}}$ & 2000 & $2000^{\mathrm{b}}$ \\
\hline
\end{tabular}

Notes. Radiation dose assumes a $1 \mu \mathrm{m}$ irradiated layer and density equivalent to that of methanol. A dose of $100 \mathrm{eV} / 16$ amu is statistically sufficient to have broken every bond in the irradiated layer at least once.

a $150 \mathrm{~K}$ was used instead of $120 \mathrm{~K}$ for the methanol.

${ }^{\mathrm{b}}$ An additional irradiation period of about $44 \mathrm{hr}$ (a little over $1000 \mathrm{eV} / 16 \mathrm{amu}$ ) at $142 \mathrm{~K}$ was performed after the $120 \mathrm{~K}$ irradiation for Round 3: Non-specular experiments.

pure methanol was analyzed to check our method against the prior published data of Brunetto et al. (2006). Ice mixtures were prepared in the gas phase in a dosing manifold, then leaked into the main chamber at a constant rate through capillary tubes pointed at the center of the sample surface from less than one centimeter distance. Experiments rich in water-ice (comet-like relative abundances) were found to have similar chemical behavior under irradiation to water-poor mixtures, but with poorer signal-to-noise on non-water-ice spectral features of interest. Therefore, water was viewed as a dilutant in the present work, and the relative amount of water in the ices was decreased compared to what has been seen from comets. This would be unlikely to affect the direction of the final slope of the spectra, but may affect the absolute value of the slope or the rate at which the final slope is obtained. Table 1 lists key experimental parameters.

After deposition, a $1 \mathrm{~cm}^{2}$ region of the ices was irradiated at $50 \mathrm{~K}$ with a beam of $10 \mathrm{keV}$ electrons (the electron current was measured periodically and adjusted to about $0.5 \mu \mathrm{Amps}$ as needed). While irradiation in space consists of a variety of particles (i.e., photons, ions, and electrons) much of the damage is stimulated by low-energy secondary electrons; therefore, we employ electrons here. We expect that ion irradiation would produce similar results. On the other hand, relatively lowenergy photons (i.e., VUV) may not provide sufficient energy per particle to stimulate all of the damage observed with higher energy particles. Direct comparison of results with different irradiation sources will not be examined further here. Electrons of $10 \mathrm{keV}$ will penetrate about $1-2 \mu \mathrm{m}$ into an ice film, forming irradiation products only within this range. The ices were then warmed at $0.5 \mathrm{Kminutes}^{-1}$ to $120 \mathrm{~K}$ and held at that temperature under continued irradiation (see Table 1). This process was intended to simulate a plausible history for an object that formed an irradiation crust at low temperatures at about 20 au from the Sun, and eventually moved inward to the warmer present-day orbital distance of Jupiter, where irradiation continued. After irradiation had been concluded at $120 \mathrm{~K}$, the thin film samples were heated to $300 \mathrm{~K}$ at $1 \mathrm{~K}$ minutes $^{-1}$, while continuing data collection. In the non-specular experiments, the sample was additionally heated to $142 \mathrm{~K}$ (after the hold at $120 \mathrm{~K}$ ) and held under continued irradiation for a couple days. This step was added to investigate how sensitive the system was to the final irradiation temperature, while still remaining below the temperature at which rapid loss of unreacted ices would occur. Irradiation was concluded at $142 \mathrm{~K}$, but spectra collected during the process of slowly heating through the rapid sublimation regime (approximately 150-200 K) are also shown below.

Note that, while pure $\mathrm{H}_{2} \mathrm{~S}$ and/or pure $\mathrm{NH}_{3}$ are highly unstable to sublimation loss at $120 \mathrm{~K}$ at the pressures used here, in the case of an intimate mixture with stable components like $\mathrm{CH}_{3} \mathrm{OH}, \mathrm{H}_{2} \mathrm{O}$, and non-volatile irradiation products, very little loss of $\mathrm{H}_{2} \mathrm{~S}$ or $\mathrm{NH}_{3}$ would be expected at this point in the experiment (e.g., Collings et al. 2004). Indeed, there was only a moderate rise in chamber pressure during heating to $120 \mathrm{~K}$ (mostly from $\mathrm{CO}_{2}$ ) and substantial $\mathrm{H}_{2} \mathrm{~S}$ and $\mathrm{NH}_{3}$ were lost concurrent with the $\mathrm{H}_{2} \mathrm{O}$ and $\mathrm{CH}_{3} \mathrm{OH}$ only at temperatures around $150 \mathrm{~K}$.

The specular-geometry, "thin film" experiments were also used to investigate the chemical constituents formed during irradiation and thermal processing by observation in the midinfrared; these results have been reported by Mahjoub et al. (2016) and highlight the critical role that sulfur plays in altering the chemistry of the irradiated ices. Additional sulfur-containing compounds not identified in the mid-infrared were identified by mass spectrometry while heating the samples to room temperature (Mahjoub et al. 2017). Thicknesses (and uncertainties) reported in Table 1 were extrapolated from the thin film results of Mahjoub et al. (2016) based on the known increase in quantity of gas dosed here.

\section{Data Processing}

The data output from the VNIR spectrometer was raw photon counts versus wavelength, producing spectra that were a convolution of the source spectrum, the detector response function, and the spectra of components in the beam path. In order to remove instrument contributions from the data and isolate the spectra alone, data for the specular reflectance 
geometry were corrected to reflectance, $R$, using:

$$
R=S \frac{g^{*}}{g_{0}},
$$

where $S$ is the raw counts, $g^{*}$ is a reference gold spectrum, and $g_{0}$ is the raw counts collected from the substrate gold mirror before sample deposition. Spectra in the absolute calibration showed distinct curvature around $600 \mathrm{~nm}$, consistent with a contribution from gold's spectral slope and implying that the ice films were not optically thick. Gold has a spectrum that is essentially flat (slope $4.6 \times 10^{-5}$ per nm) between 650 and $1650 \mathrm{~nm}$. The absolute reflectance spectra were then also ratioed to gold and/or to pre-irradiation ice to accentuate spectral changes with each experimental step, using a comparison in relative reflectance. The thick ice mixtures did not have a suitable reference spectrum available to perform the correction to absolute radiation above. In lieu of this, the data for these experiments were referenced only to their unirradiated mixed-ice (shortly before irradiation began).

\section{Results and Discussion}

\subsection{Methanol Thin Ice and Discussion of Uncertainty}

Selected spectra from the methanol irradiation at $50 \mathrm{~K}$ are shown in Figure 1. Figure 1(a) shows the raw signals from each of the two spectrometer ranges, while the remaining panels in Figure 1 show three ways of processing the data to obtain meaningful spectra. Each of these data processing methodsabsolute and relative reflectance to different standards-has its advantages. Since the signal entering the spectrometer varied by several orders of magnitude in some experiments, the relative contribution from the underlying mirror could not be assumed constant. Correcting for the instrument contributions allows visual examination of the changing contribution from the gold mirror substrate; however, it makes comparison with astronomical and other lab spectra more difficult. Referencing the spectra to a gold mirror substrate (i.e., assuming the substrate was providing a constant contribution to the spectra throughout the experiment) is how lab work is often processed (e.g., Brunetto et al. 2006), thus the inclusion of this method in Figure 1(c). Finally, since deposition of some of the ices took significant amounts of time and changed the total signals in some cases by orders of magnitude, there was a very real possibility of changing contributions from the instrument as well. Referencing the spectra during the irradiation and heating phases to the spectrum of the ice shortly before irradiation (see Figure 1(d)) effectively excluded the above factors and showed the changes in the spectra occurring after irradiation began. The downside of referencing to the ice itself is that it divides out any inherent spectral features or slope of the unirradiated mixed ice, showing only the changes from that point. Since all three processing approaches have value, all three are included for each experiment below (when possible).

Extended characterization of the instrument stability over periods of 8 and 12 days have been conducted and reported elsewhere (Poston et al. 2017). The VNIR detector was found to drift within a $10 \%$ range, including changes in the slope of the spectra. The general trend was that the spectra would be stable within a few percent for tens of hours, then drift over a few hours to a new state of relative stability, and so on. The most stable results were obtained for the spectral range from the mid-400 s to the mid-1000s of nanometers. Within this range the instrument response tended to drift smoothly, changing the entire spectrum in a uniform fashion, while the wings of the range drifted independently.

The NIR detector range from 700 to $1650 \mathrm{~nm}$ was also characterized over 12 days, concurrent with the VNIR characterization, but with less temporal resolution. The overall bounds of the drift were similar to the VNIR; the response varied within a $10 \%$ range. The lack of temporal resolution excluded characterizing how quickly the response shifted, but shifts were well-correlated with the shifts happening in the VNIR range. Similar to the VNIR, the NIR also performed the worst on the wings of its response range, with the best performance from the mid- $900 \mathrm{~s}$ to the mid- $1400 \mathrm{~s}$ of nanometers.

Normalized spectra from the methanol irradiation experiment are shown in Figure 2. Both detectors performed well in an overlapping range from the mid-900s to the mid-1000s, so normalization was conducted at $950 \mathrm{~nm}$. For clarity, the most poorly performing ranges of each detector range were omitted. The unirradiated methanol ice presented a slightly red slope after deposition at $50 \mathrm{~K}$. Irradiation created a steeper red slope, as did heating to $150 \mathrm{~K}$. An hour of irradiation at $150 \mathrm{~K}$ may have further steepened the slope, but the effect was too small to claim with confidence.

Methanol was analyzed primarily to test how the present experiment compared to previous work by other investigators under similar conditions. However, several notable differences exist between our work and that of Brunetto et al. (2006), including (1) thickness of ice film-our work used a methanol film about twice as thick as that of Brunetto et al.; (2) radiation type-Brunetto et al. used ions while our work used electrons; (3) observation geometry-Brunetto et al. used a diffuse reflection geometry and a diffuse substrate while our work primarily used a specular geometry and a mirror substrate; and (4) ice film temperature-Brunetto et al. used a temperature of $77 \mathrm{~K}$ while our work used a temperature of $50 \mathrm{~K}$. Despite these differences in experimental approach, the overall results here were similar to those reported by Brunetto et al.; upon irradiation, both experiments showed substantial reddening (positive spectral slope). The numerical values of the normalized slopes match quite well between the last spectrum in each work; however, the temperature and irradiation dose were not the same in each case, so it is unclear if the numerical comparison is meaningful or mere coincidence. In the present work, heating from 50 to $150 \mathrm{~K}$ resulted in substantial reddening, establishing a dependence on temperature. Having shown results consistent with this previous work, we proceeded to the mixed ices.

\subsection{Mixed Ice Thin Film Results}

Selected spectra from the thin $(2 \mu \mathrm{m})$ "3-ice" and "3-ice $+\mathrm{H}_{2} \mathrm{~S}$ " experiments in the specular geometry are shown in Figures 3-5 (the 3-ice $+\mathrm{H}_{2} \mathrm{~S}$ experiment was performed twice in these conditions). The conditions for these experiments were essentially identical and spectra were acquired at the same stages in each experiment for each ice. All unirradiated ices showed structure shortward of $600 \mathrm{~nm}$. This structure may have been due to refractive index matching between the ice and the gold mirror (the wavelength-dependent real refractive index of gold is similar to that of ice in this wavelength range; Johnson \& Christy 1972), resulting in wavelength-dependent absorption at the ice-gold interface. Since the refractive indices of our 

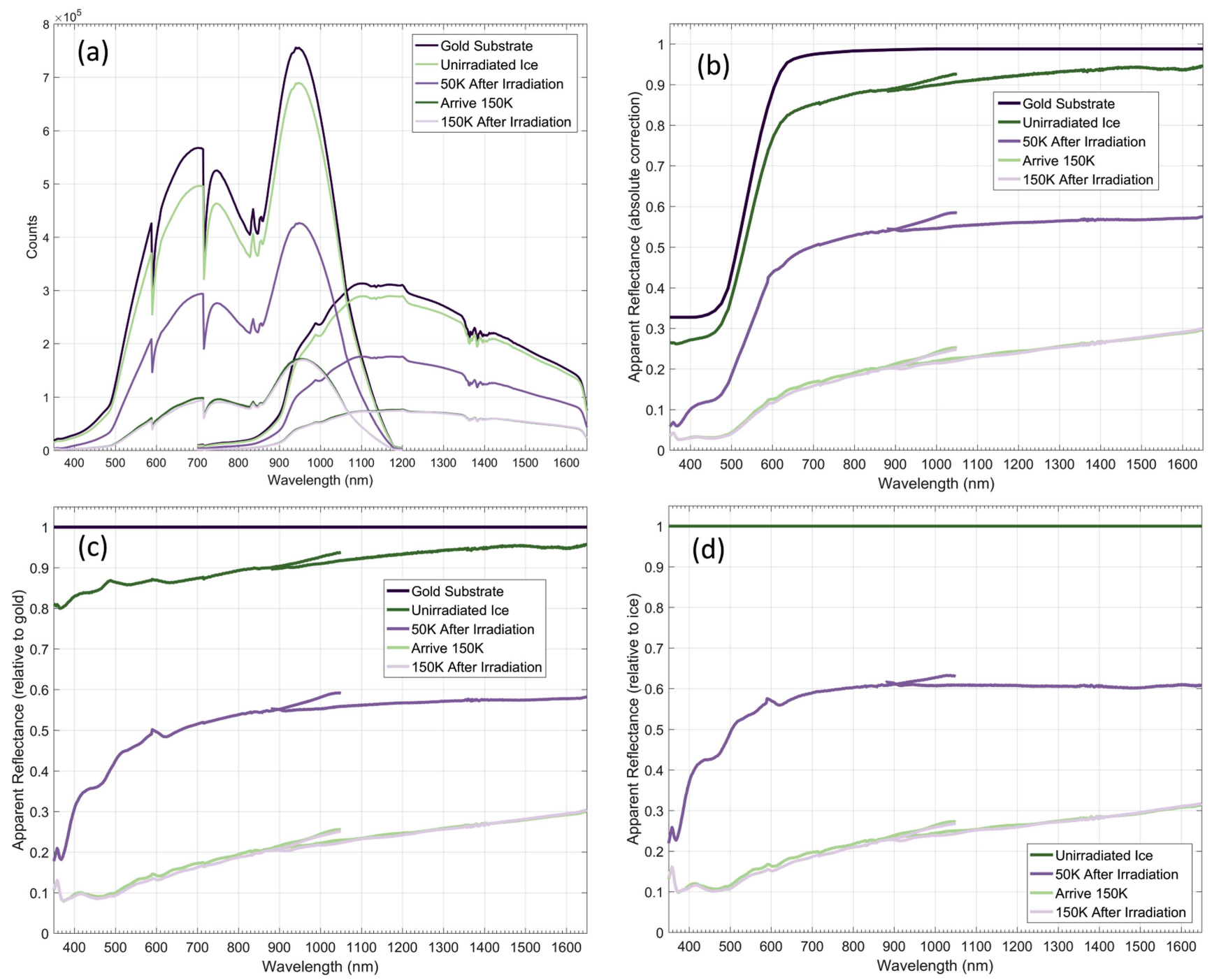

Figure 1. Selected spectra from the $2 \mu \mathrm{m}$ thick methanol irradiation experiment. The signal from both detectors is shown, including the region of overlap. (a) Raw signals received by the detector; (b) absolute reflectance, corrected for instrument contributions by ratio to the gold reference and then correction for the spectral properties of gold; (c) relative reflectance, referenced to the gold mirror shortly before deposition; and (d) referenced to the ice shortly before irradiation began. Relative uncertainty was larger in all spectra shortward of $650 \mathrm{~nm}$ due to photon absorption by the gold mirror substrate. Order-sorting filter insertion is clearly visible in (a) at about 590 and $720 \mathrm{~nm}$ and often resulted in narrow artifacts in the data at these wavelengths, such as is seen in (c). Note that the "Arrive $150 \mathrm{~K}$ " and " $150 \mathrm{~K}$ After Irradiation" sample data in this experiment are nearly indistinguishable in all panels.

intimate mixtures of ices were not known, analysis of the contribution (if any) from refractive index matching was not further examined. In addition to possible refractive index matching, the distinct feature at about $490 \mathrm{~nm}$ in the unirradiated ice was a known artifact of the setup. This artifact corresponded to the kink seen in Figure 1(a) at this same wavelength and tended to show up most prominently in cases of lower counts (as will be seen in the next section).

Slopes for both mixed ices reddened and the total signal decreased with irradiation, particularly in the visible wavelengths. Slope and signal changes at $50 \mathrm{~K}$ were larger for the 3 -ice, particularly in the NIR where the 3-ice data showed a positive spectral slope while the 3 -ice $+\mathrm{H}_{2} \mathrm{~S}$ ice data remained relatively flat. Heating from 50 to $120 \mathrm{~K}$ did not appreciably change the slope for either ice mixture. In addition to the changes in slopes and signals, strong absorption bands centered at about 410, 600, and $900 \mathrm{~nm}$ appeared in both 3 -ice $+\mathrm{H}_{2} \mathrm{~S}$ experiments, but not in the 3-ice experiment. These bands appeared to have deepened during irradiation at $120 \mathrm{~K}$.

Ending irradiation and heating $\left(0.5 \mathrm{~K} \mathrm{minutes}^{-1}\right)$ through the mid $150 \mathrm{~K}$ temperature range first steepened the slope and reduced the total signal, then destroyed the bands for the 3 -ice $+\mathrm{H}_{2} \mathrm{~S}$, while having very little effect on the 3-ice (see Figure 6). Slopes then decreased with additional heating until at $180 \mathrm{~K}$ the 3 -ice $+\mathrm{H}_{2} \mathrm{~S}$ slope was just slightly steeper than that of the 3-ice. When temperatures reached the upper $180 \mathrm{~K}$ range, the total signal for both mixtures rapidly decreased with heating until stabilizing at about $200 \mathrm{~K}$ at about one-tenth of the signal as from the gold mirror. The signals and slopes remained relatively constant until the low $240 \mathrm{~K}$ range, where signal gradually increased with temperature. Absolute slope remained nearly constant while the signal increased; therefore, the normalized slope decreased above $240 \mathrm{~K}$. Slopes were redder and the signal higher for the 3-ice than for the 3 -ice $+\mathrm{H}_{2} \mathrm{~S}$ 

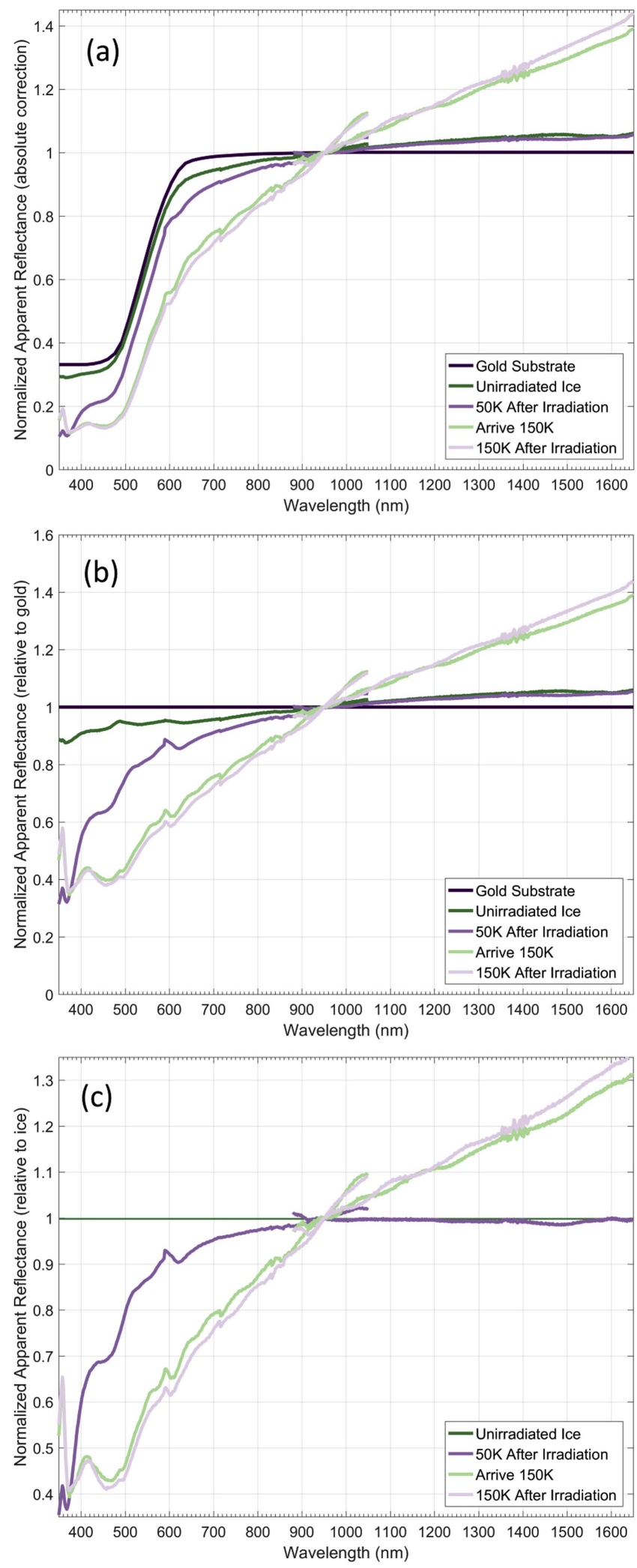

Figure 2. Selected spectra from the $2 \mu \mathrm{m}$ thick methanol irradiation experiment. Spectra were normalized at $950 \mathrm{~nm}$ and some overlap between detectors is shown. (a) Corrected for instrument contributions; (b) referenced to the gold mirror shortly before deposition; and (c) referenced to the ice shortly before irradiation began. Details are the same as those in Figure 2.

above $200 \mathrm{~K}$, making about $160-190 \mathrm{~K}$ the only temperature range where slopes were observed to be redder for the irradiated 3-ice $+\mathrm{H}_{2} \mathrm{~S}$ mixture, for the thin films.

\subsection{Mixed Ice Thick Film Results}

Uncertainty in the thin film results stemmed from the potential for refractive index matching and changes in the relative contribution of the gold mirror substrate to the spectra as the scattering properties of the film changed with irradiation and heating. For example, if submicron carbon particles formed during the irradiation, they could serve as Raleigh and Mie scattering centers similar to submicron dark materials in silicate space weathering (for example, Pieters \& Noble 2016). Therefore, experiments with thicker ice films were conducted in an attempt to assess changes in the VNIR spectra with a lower and less variable contribution from the substrate.

The "thick ice" experiments were conducted in two geometries: the same specular geometry as the $2 \mu \mathrm{m}$ "thin ice" experiments, and a non-specular geometry where the detector collection mirror was located nearly orthogonal to the specular reflection path. Thicknesses are reported in Table 1 and ranged from $8 \mu \mathrm{m}$ to about $200 \mu \mathrm{m}$. Each stage (i.e., deposition, irradiation at $50 \mathrm{~K}$, and irradiation at $120 \mathrm{~K}$ ) of the specular-geometry thick ice experiments was continued until the spectra appeared to have reached a steady state. The 3-ice was more scattering than the 3-ice $+\mathrm{H}_{2} \mathrm{~S}$ mixed ice, so steady-state deposition was achieved with a significantly thinner film. While thickness may have contributed to the observed results, taking the irradiation to spectral equilibrium is probably most relevant to real solar system bodies, which were exposed to an excess of radiation.

Selected spectra from the thick 3-ice and 3-ice $+\mathrm{H}_{2} \mathrm{~S}$ experiments in the specular geometry are shown in Figures 7 and 8. These experiments were conducted over several weeks, over which the signal exiting the chamber varied by several orders of magnitude, requiring substantial increases in integration time. Lacking the ability to collect a new reference spectrum during the experiment, significant uncertainty was introduced by the long duration of the experiments. As was discussed above, the greatest uncertainty existed at the short and long wavelength ends of the spectra. Since long integration times were required for the VNIR detector, the low-count region from 350 to $450 \mathrm{~nm}$ was not collected for any thick ice experiments. In addition, the sharp experimental artifact at $490 \mathrm{~nm}$ dominated the 3-ice spectra shortward of $550 \mathrm{~nm}$ (Figure 7). This artifact was prominent in this specific experiment because of the following factors: (1) relatively low total counts for both the reference (the unirradiated ice) and the irradiated ice, and (2) a relatively small change in total counts after irradiation. Together, these resulted in relatively poor signal-to-noise shortward of $550 \mathrm{~nm}$. The 3-ice $+\mathrm{H}_{2} \mathrm{~S}$ (Figure 8) also had an artifact at $490 \mathrm{~nm}$, but it was small compared to the change in signal during irradiation and is difficult to see in the plot. Finally, there was evidence of significant drift longward of $1400 \mathrm{~nm}$ during the deposition phase of the thick ice experiments. Both of these ranges have been shaded gray in the figures and were not included in the following interpretations.

Given all the uncertainties, the data were, nevertheless, remarkably consistent from spectrum to spectrum. Similar to the thin ice results above, the thick 3 -ice results contained no significant features. (The shallow dip centered at $800 \mathrm{~nm}$ may have been a feature, but was less than the uncertainty for this trial and was not seen in any of the other 3-ice experiments, so it was not interpreted as a feature.) However, in contrast to the thin ices, the thick 3-ice results lacked any clear spectral slope. This could indicate that the ultimate state of the 3-ice, when 

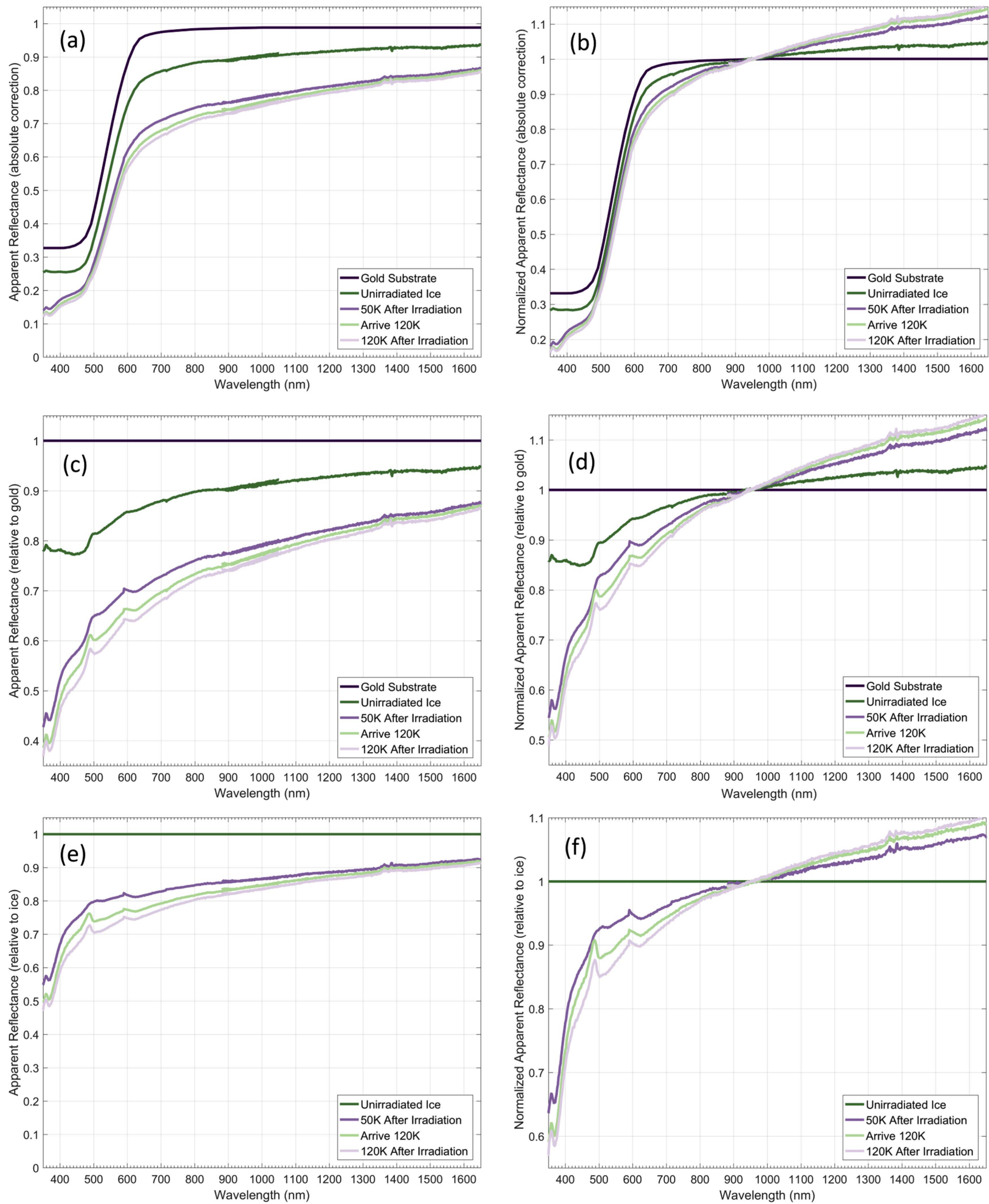

Figure 3. Selected spectra from the $2 \mu \mathrm{m}$ thick, 3-ice (water:methanol:ammonia, 1:2:2) irradiation experiment. Figures in the right column were normalized at 950 nm and some overlap between detectors is shown. ((a) and (b)) Corrected for instrument contributions; ((c) and (d)) referenced to the gold mirror shortly before deposition, and ((e) and (f)) referenced to the ice shortly before irradiation began. Details are the same as those in Figure 2.

irradiated to equilibrium, is a neutral slope (similar to that of hydrocarbon mixtures, see Andronico et al. 1987). However, the total dose at $50 \mathrm{~K}$ was similar in both the thin and thick specular-geometry experiments, so this is probably not the correct interpretation. Another possibility is that any absorption or scattering due to irradiation products was too weak to be seen overlain on tens of microns of highly scattering unirradiated ice. The 3-ice $+\mathrm{H}_{2} \mathrm{~S}$ results exhibited both a steep, 

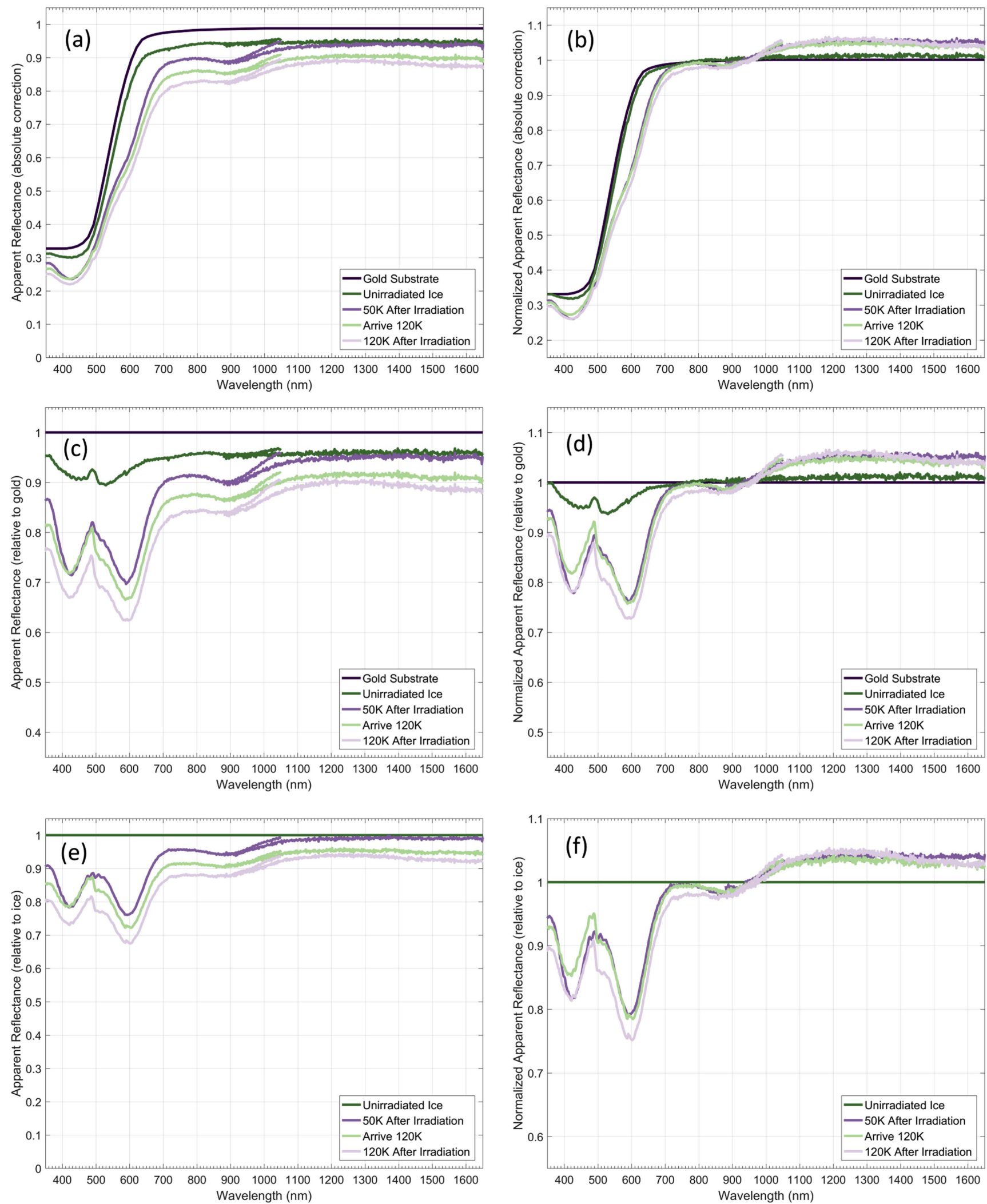

Figure 4. Selected spectra from the $2 \mu \mathrm{m}$ thick, 3 -ice $+\mathrm{H}_{2} \mathrm{~S}$ (water:methanol:ammonia:hydrogen sulfide, 1:3:3:3) irradiation experiment. Figures in the right column were normalized at $950 \mathrm{~nm}$ and some overlap between detectors is shown. ((a) and (b)) Corrected for instrument contributions, ((c) and (d)) referenced to the gold mirror shortly before deposition, and ((e) and (f)) referenced to the ice shortly before irradiation began. Details are the same as those in Figure 2.

red slope and possible absorption features at 620 and $900 \mathrm{~nm}$. The downturn in the spectra shortward of $500 \mathrm{~nm}$ was consistent with the possible feature at $410 \mathrm{~nm}$ that was seen in the thin ice experiments above.
Selected spectra from the thick 3-ice and 3-ice $+\mathrm{H}_{2} \mathrm{~S}$ experiments in the non-specular geometry are shown in Figures 9 and 10. Dosing in these two experiments was performed to an approximately equivalent signal on the VNIR 

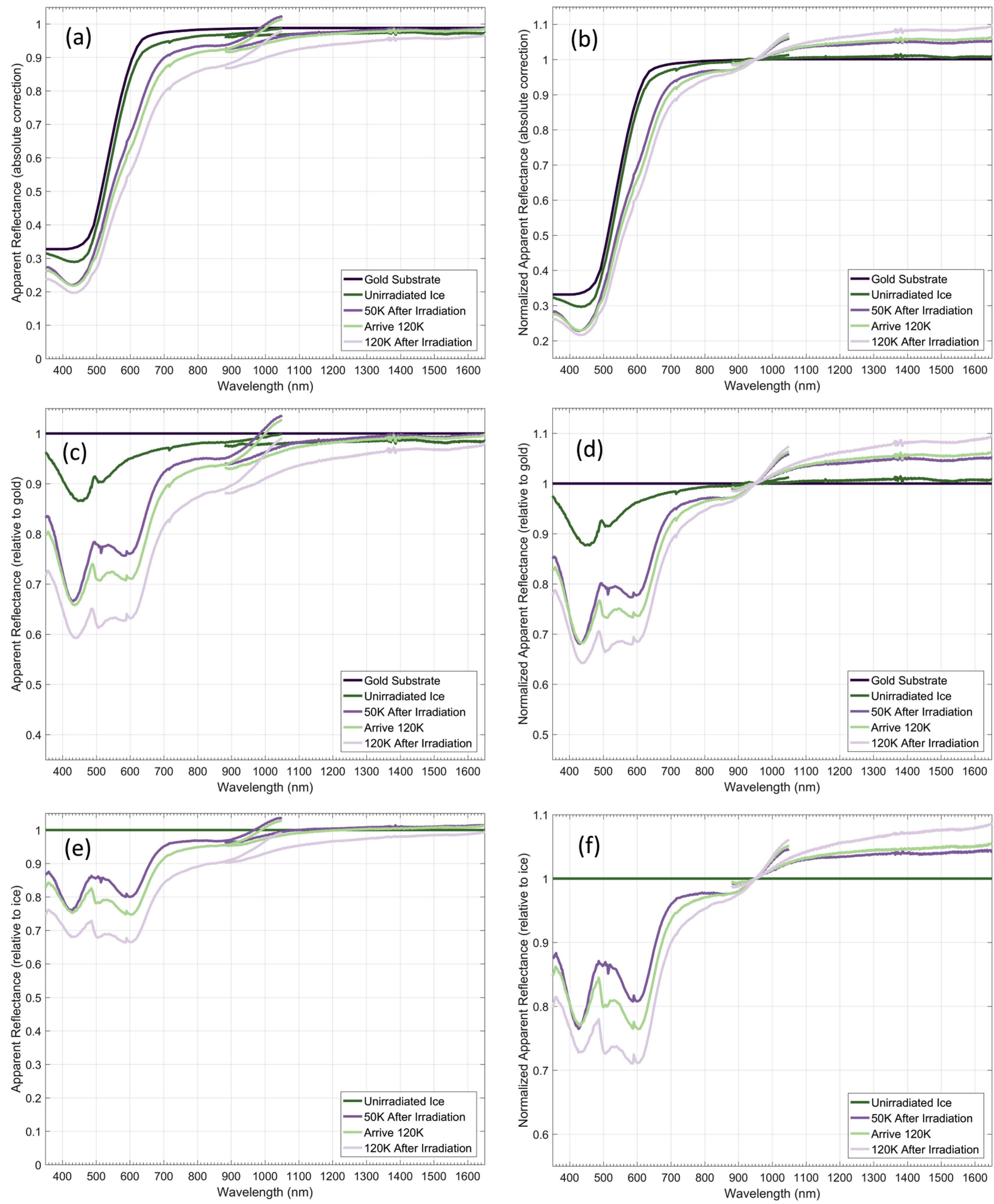

Figure 5. Selected spectra from the repeat $2 \mu \mathrm{m}$ thick 3-ice $+\mathrm{H}_{2} \mathrm{~S}$ (water:methanol:ammonia:hydrogen sulfide, 1:3:3:3) irradiation experiment. Figures in the right column were normalized at $950 \mathrm{~nm}$ and some overlap between detectors is shown. ((a) and (b)) Corrected for instrument contributions; ((c) and (d)) referenced to the gold mirror shortly before deposition, and ((e) and (f)) referenced to the ice shortly before irradiation began. Details are the same as those in Figure 2 .

spectrometer. The 3-ice $+\mathrm{H}_{2} \mathrm{~S}$ required about eight times greater thickness to reach equivalent signal, which is interpreted as meaning the 3 -ice $+\mathrm{H}_{2} \mathrm{~S}$ film was less scattering than the 3 -ice (consistent with greater thickness being required to obscure the gold mirror in the previous round of experiments). Slope and feature results from 550 to $1400 \mathrm{~nm}$ during the irradiation at 50 and $120 \mathrm{~K}$ were in good agreement with those of the thin ices. Specifically, the 3-ice showed 

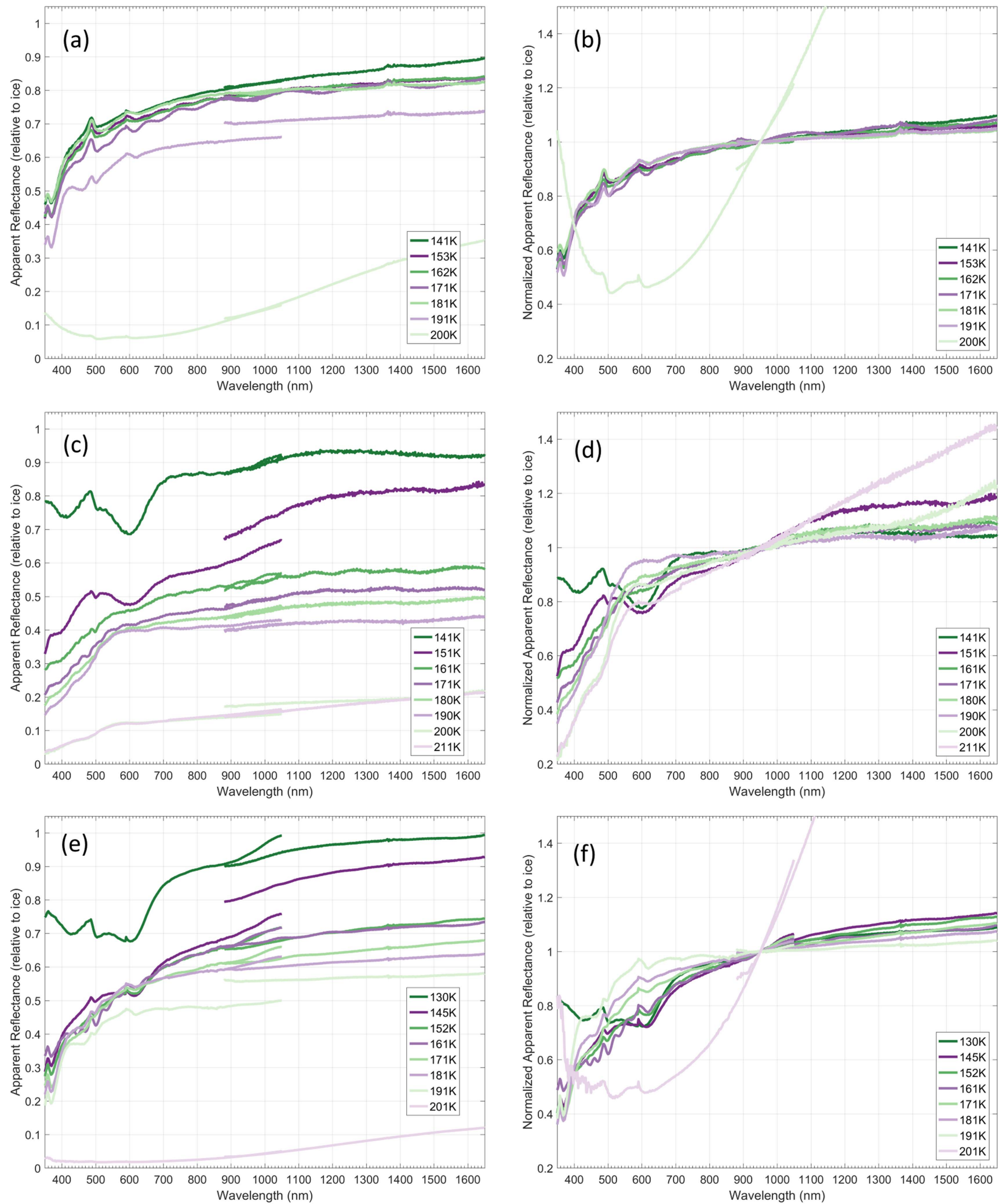

Figure 6. Spectra collected while heating $2 \mu \mathrm{m}$ thick samples at a rate of $0.5 \mathrm{~K}_{\text {minutes }}{ }^{-1}$. Spectra progress from dark $(130 \mathrm{~K})$ to light (200 K). Figures in the right column were normalized at $950 \mathrm{~nm}$ and some overlap between detectors is shown. ((a) and (b)) 3-ice; ((c) and (d)) 3-ice $+\mathrm{H}_{2} \mathrm{~S}$; and ((e) and (f)) repeat 3-ice $+\mathrm{H}_{2} \mathrm{~S}$. Only the data referenced to the unirradiated ice shortly before irradiation are shown. Note that some of the materials were lost to sublimation upon heating, generating appreciable changes in spectra.

featureless spectra and a red slope of similar magnitude to the thin ices, and the 3 -ice $+\mathrm{H}_{2} \mathrm{~S}$ showed features at about 620 and $900 \mathrm{~nm}$ and an underlying red slope. However, in the non-specular case, both the underlying slope and a fit to the data with the absorption peaks included resulted in a steeper slope for the 3 -ice $+\mathrm{H}_{2} \mathrm{~S}$. Heating from 50 to $120 \mathrm{~K}$ under 

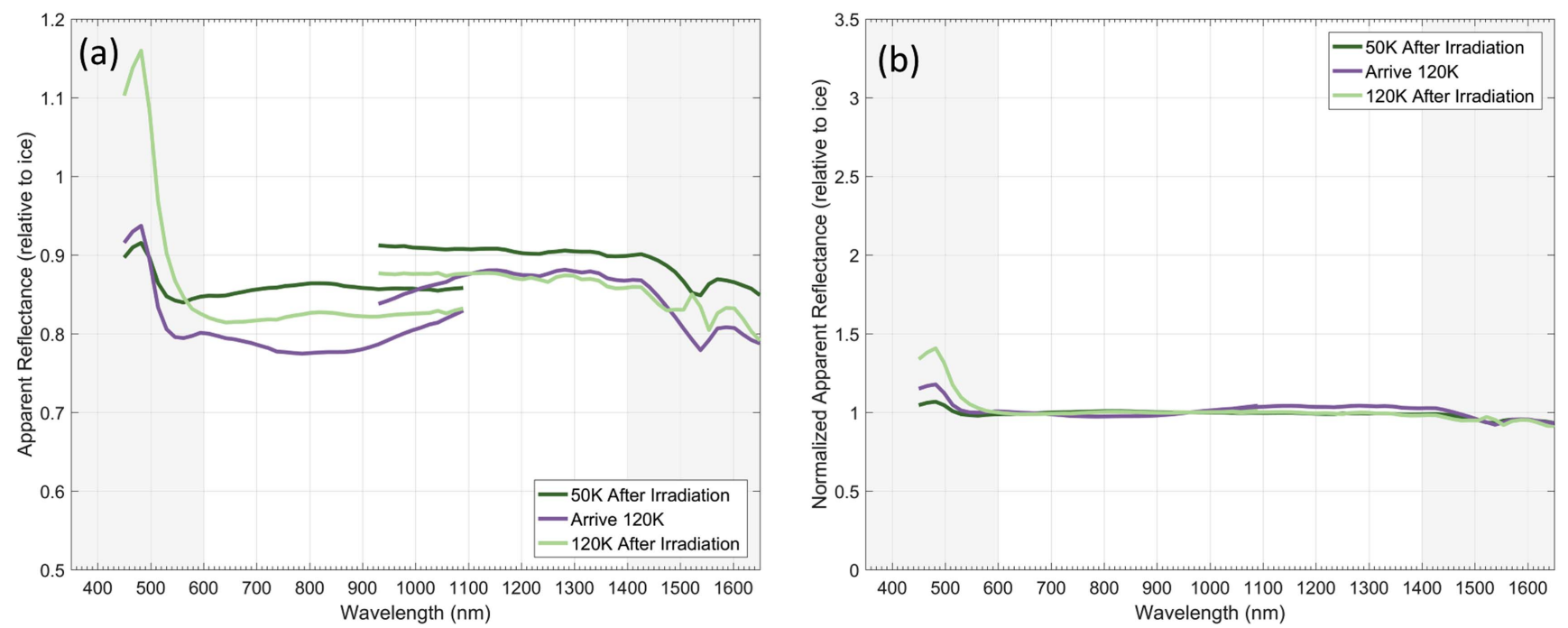

Figure 7. Selected spectra from the $40 \mu \mathrm{m}$ thick, 3-ice (water:methanol:ammonia, 1:2:2) irradiation experiment. Irradiation in this experiment was continued until the spectra showed apparent equilibrium. (a) Apparent reflectance relative to unirradiated ice shortly before irradiation, and (b) spectra normalized at $950 \mathrm{~nm}$. Gray background indicates regions of lower confidence in the data (see the text).
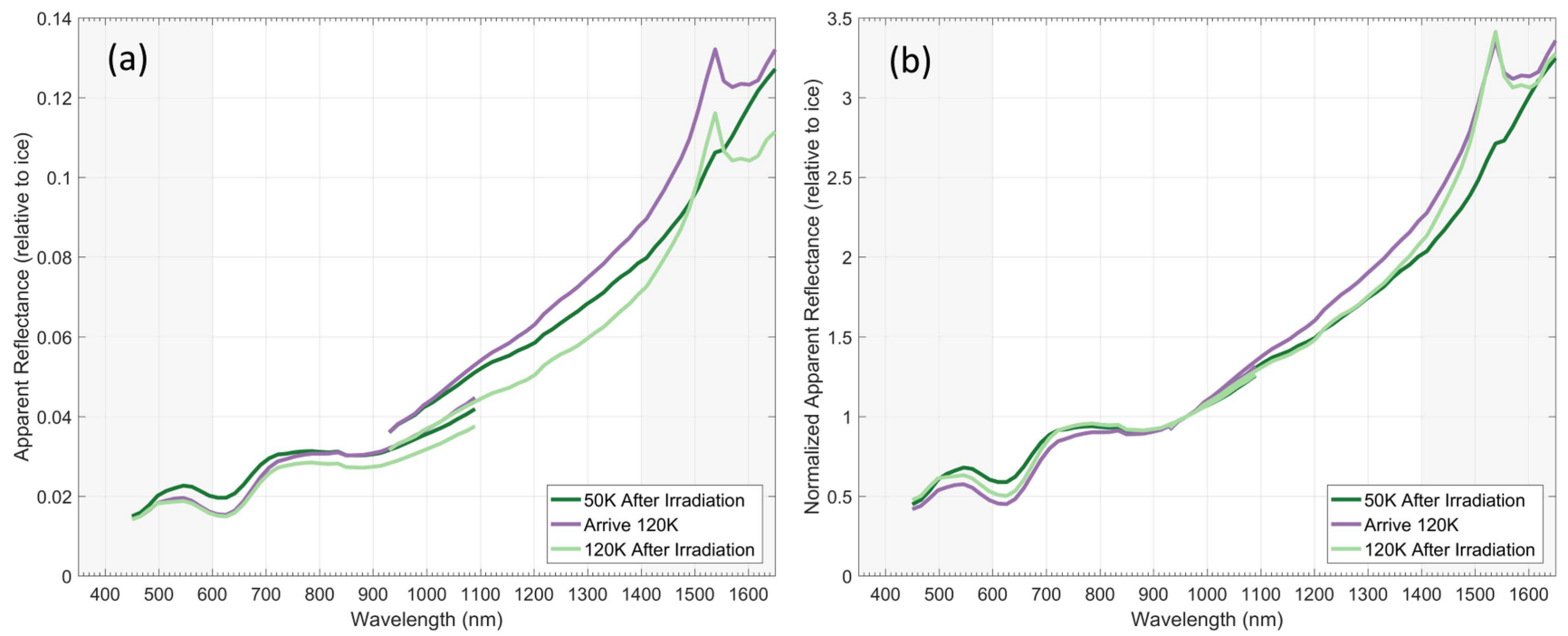

Figure 8. Selected spectra from the $200 \mu \mathrm{m}$ thick, 3-ice $+\mathrm{H}_{2} \mathrm{~S}$ (water:methanol:ammonia:hydrogen sulfide, 1:3:3:3) irradiation experiment. Irradiation in this experiment was continued until the spectra showed apparent equilibrium. (a) Apparent reflectance relative to unirradiated ice shortly before irradiation and (b) spectra normalized at $950 \mathrm{~nm}$. Gray background indicates regions of lower confidence in the data (see the text).

irradiation steepened the slopes for both samples, and may have deepened the bands for the 3-ice $+\mathrm{H}_{2} \mathrm{~S}$, while further irradiation at $120 \mathrm{~K}$ had little effect. Heating to $142 \mathrm{~K}$ and holding under continued irradiation had a negligible effect on the spectra. However, after irradiation was concluded at $142 \mathrm{~K}$ the features on the 3-ice $+\mathrm{H}_{2} \mathrm{~S}$ were lost over time, with heating to $150 \mathrm{~K}$ increasing the rate of feature loss. Similar to the thin films, the slopes decreased with heating above $150 \mathrm{~K}$, with the 3 -ice $+\mathrm{H}_{2} \mathrm{~S}$ slope becoming steeper than the 3-ice during the hold at $167 \mathrm{~K}$. Slopes increased gradually, to a maximum value around $290 \mathrm{~K}$, then decreased slightly as temperature stabilized at $300 \mathrm{~K}$. The magnitude of slope changes while heating from 170 to $300 \mathrm{~K}$ in the non-specular geometry was much smaller than that in the thin-film specular geometry experiments.

Trends in total signal up to $167 \mathrm{~K}$ were generally the opposite of the thin ice experiments in the specular geometry. Both mixture compositions increased in total signal with irradiation, with 3-ice $+\mathrm{H}_{2} \mathrm{~S}$ exhibiting an increase of up to a factor of seven. This was consistent with the behavior seen between deposition in the two geometries and indicated that changes in scattering behavior were a key effect of irradiation. Total signal changes above $167 \mathrm{~K}$ did trend in the same direction in both geometries, although the magnitude of changes was much smaller in the thick ice non-specular geometry. Total signal for both mixtures was stable from 167 to $180 \mathrm{~K}$, then began to decrease. Signal decrease slowed at about $200 \mathrm{~K}$ then sped up again above $240 \mathrm{~K}$ for the 3 -ice $+\mathrm{H}_{2} \mathrm{~S}$, while it was relatively constant for the 3 -ice. At about $270 \mathrm{~K}$, trends changed for both samples: for the 3 -ice $+\mathrm{H}_{2} \mathrm{~S}$, the signal began to increase again, while for the 3-ice the rate of signal decrease sped up. The relative magnitude of signal changes was larger in the 3-ice $+\mathrm{H}_{2} \mathrm{~S}$, though both were an order of magnitude smaller than relative changes in the thin ices in the same temperature ranges. The fact that the total signal remained relatively constant even as the ices were removed by heating indicates that spectra in the non-specular geometry experiments 

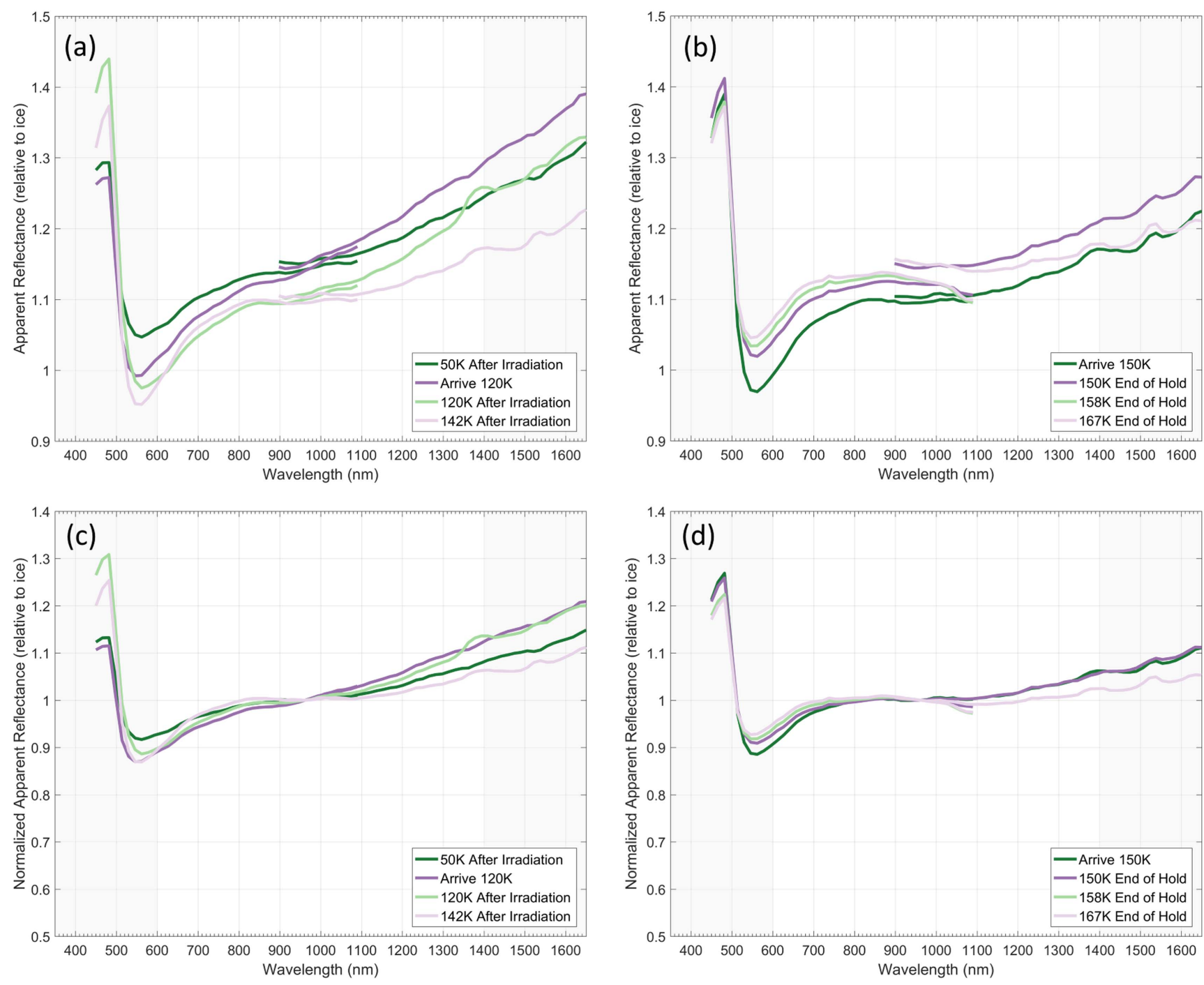

Figure 9. Selected spectra from the $8 \mu \mathrm{m}$ thick 3-ice experiment in a non-specular geometry. ((a) and (b)) Apparent reflectance relative to unirradiated ice shortly before irradiation and ((c) and (d)) same spectra normalized at $950 \mathrm{~nm}$. Due to blank issues, only spectra referenced to the ice shortly before irradiation were possible for this experiment. Data were not collected at $158 \mathrm{~K}$ for the NIR detector. Gray background indicates regions of lower confidence in the data (see the text).

were dominated by light scattered by the irradiation-produced residue(s) and that the underlying gold substrate likely contributed very little to the results.

\subsection{Assimilation of Results}

Results and uncertainties for three sets of experiments were reported above: thin specular, thick specular, and thick nonspecular; selected overlays are presented in Figure 11 and changes in slope and apparent reflectance during the irradiation phases of the experiments are summarized in Table 2. The thick ice experiments ran long enough that instrumental drift made interpretation challenging, but control experiments monitoring the instrumental drift while observing a reference standard enabled identification of which aspects could be interpreted with the highest confidence.

Methanol behaved in a manner similar to that reported by Brunetto et al. (2006), showing featureless spectra with red slopes. The 3-ice (water, methanol, and ammonia) results showed a lack of features in all three experiments, and neutralto-red slopes (depending on experiment). The 3-ice $+\mathrm{H}_{2} \mathrm{~S}$ results consistently showed red slopes underlying possible features at 620 and $900 \mathrm{~nm}$ in all three experiments, which were more pronounced for thicker ices with larger irradiation exposures, and another feature at $410 \mathrm{~nm}$ that appeared in two out of three experimental geometries. Depths for the potential features seen in the 3 -ice $+\mathrm{H}_{2} \mathrm{~S}$ experiments increased with thicker films, supporting the conclusion that these are due to irradiation products that increased in concentration with larger doses. Heating above $140 \mathrm{~K}$ and/or concluding irradiation resulted in loss of the potential absorption features and reduced the slopes of both mixtures up until about $180 \mathrm{~K}$. Over the $180 \mathrm{~K}$ to the mid $200 \mathrm{~K}$ range, both specular and non-specular geometry experiments showed decreasing signal indicating an unambiguous darkening of the samples.

The potential $410 \mathrm{~nm}$ feature may be due to $S_{3}$ (Meyer 1976), and may have contributions from refractive index matching, as was discussed above. The $620 \mathrm{~nm}$ feature may be due to $S_{5}$ (Meyer 1976). $S_{4}$ is thought to absorb at $510 \mathrm{~nm}$ (Meyer 1976), which was not seen here. The feature at $900 \mathrm{~nm}$ has not been predicted for any pure sulfur chain or ring, but we posit that it may be due to a sulfur-carbon or sulfur-nitrogen 

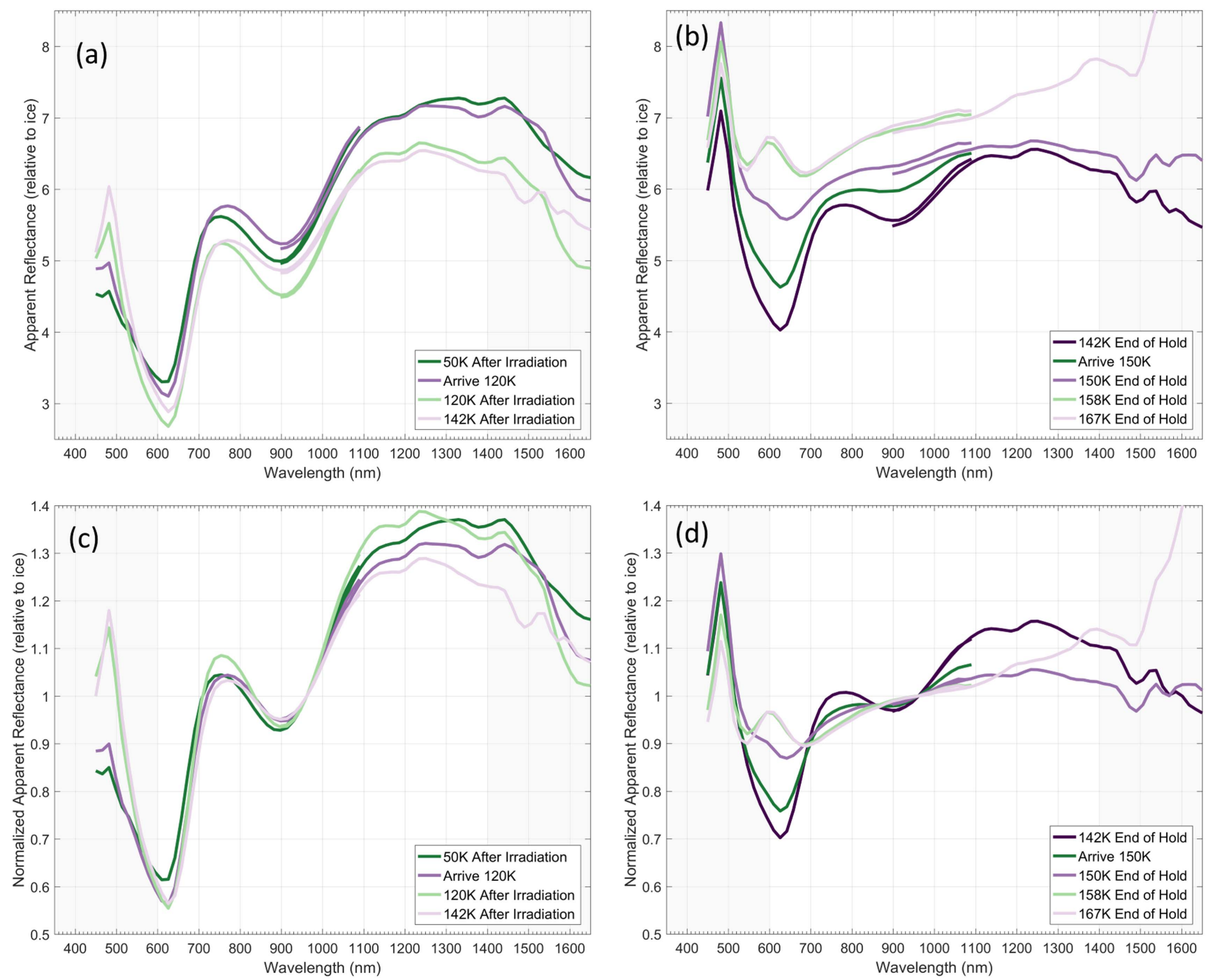

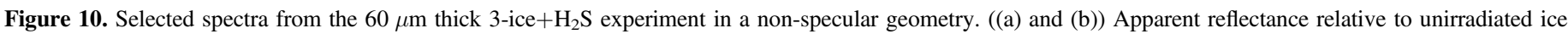

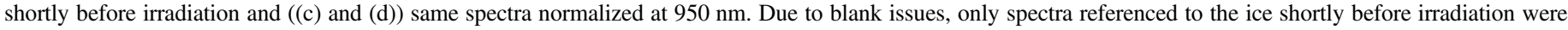

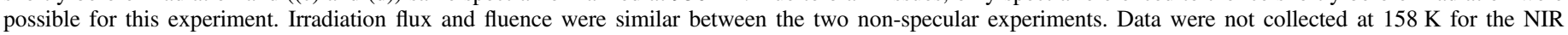
detector. Gray background indicates regions of lower confidence in the data (see the text).

compound. For example, Lebofsky \& Fegley (1976) reported features at about 400, 600, and $900 \mathrm{~nm}$ upon deposition of $\mathrm{NH}_{4} \mathrm{HS}+\mathrm{H}_{2} \mathrm{O}$. In that work, the 400 and $600 \mathrm{~nm}$ features grew under irradiation, while the $900 \mathrm{~nm}$ feature was inconsistent, whereas, in our work, all features grew under irradiation. Lebofsky and Fegley did not uniquely assign the bands, but suggested short sulfur chains for $410 \mathrm{~nm}$, sulfur diradicals (of $\mathrm{S}_{5}-\mathrm{S}_{7}$ ) for the $620 \mathrm{~nm}$ feature, and possibly clathrate hydrates of sulfur compounds for the $900 \mathrm{~nm}$ feature. The growth of the features with irradiation and decay upon heating is consistent with these potential sources, which are unstable at warmer (i.e., non-cryogenic) temperatures.

\section{Astrophysical Implications}

\subsection{Application to KBOs and Trojans}

The primary motivation for these experiments was to test the hypothesis that sulfur-containing irradiation products present in the crust of the redder groups of Trojans/KBOs-and not in the less red groups-are the key difference necessary to produce the observed color bimodality in the two subpopulations in a "Nice model" migration scenario where Trojans and KBOs formed in the same location. For the present experiment to support this hypothesis, the following three trends should exist:

(1) Spectral slopes should be steeper in the irradiated 3 -ice $+\mathrm{H}_{2} \mathrm{~S}$ mixed ice than the 3-ice sample.

(2) Irradiated films should become less bright upon heating to Trojan-like temperatures and further irradiation.

(3) Spectral slopes should become less steep upon heating to Trojan-like temperatures and further irradiation.

The above requirements were based on telescope observations (see the Introduction and Wong et al. 2014; Wong \& Brown 2016) of Trojans and small KBOs (larger KBOs greater than $\sim 300 \mathrm{~km}$ in diameter likely underwent additional secondary processes, such as differentiation, and are less relevant to the hypothesis). Since telescope observations are generally nonspecular in geometry, the most appropriate lab data for comparison with telescopic observations were the non-specular geometry results. In this geometry, the normalized " $50 \mathrm{~K}$ after irradiation" spectra showed a steeper slope for the 3-ice $+\mathrm{H}_{2} \mathrm{~S}$ 

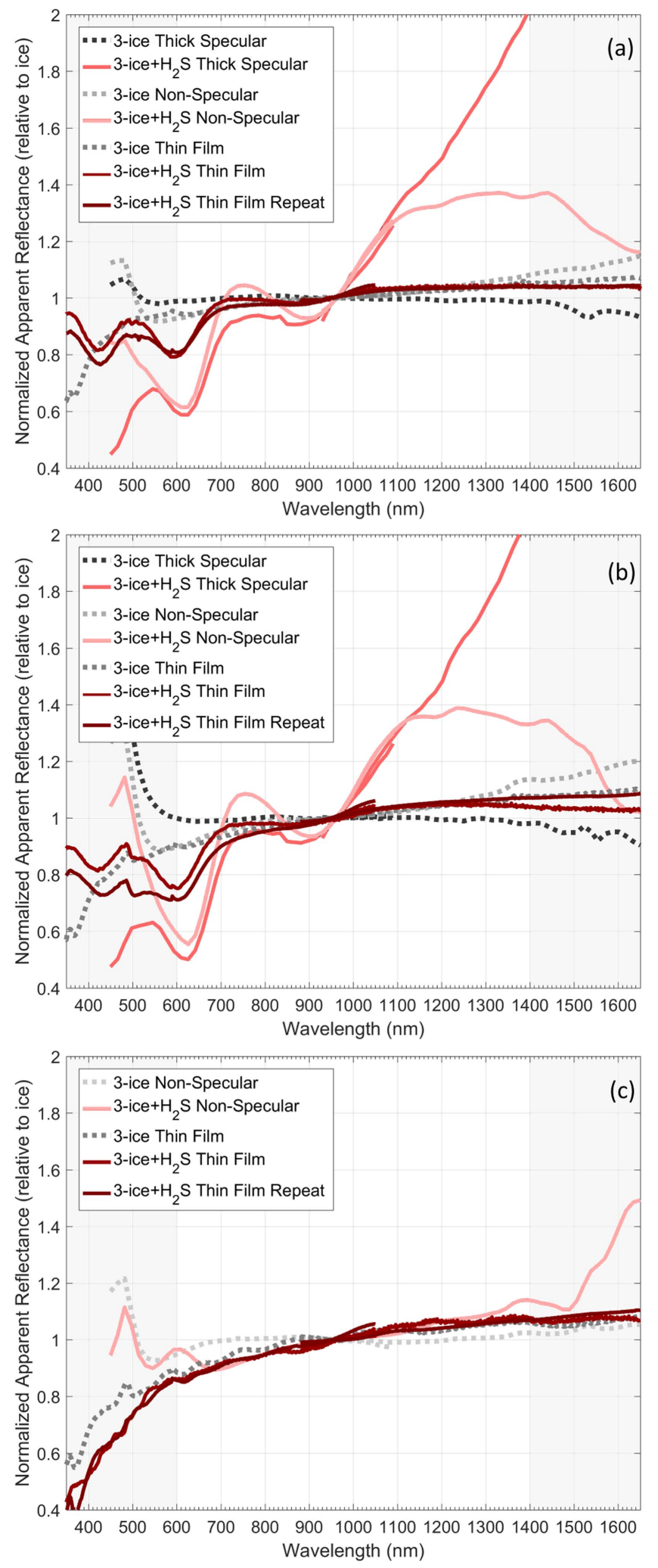

Figure 11. Overlays of normalized spectra at comparable points in each of the three rounds of experiments (normalized at $950 \mathrm{~nm}$ ). (a) At $50 \mathrm{~K}$ after irradiation; (b) at $120 \mathrm{~K}$ after irradiation; and (c) after concluding irradiation and heating to about $170 \mathrm{~K}$. Irradiation times are listed in Table 1 and experimental steps having occurred between the collection of the data in each plot are described in the text. Gray background indicates regions of lower confidence in the data (see the text). mixture (see Figure 11(a)), even when the absorption features were excluded and only the underlying slope was considered. The data at $50 \mathrm{~K}$ were most relevant to KBOs, so the above result supported trend (1) for KBOs.

The data collected with irradiation at 120 and $142 \mathrm{~K}$ were intended to represent the history of Trojan objects. These data showed essentially the same result as those at $50 \mathrm{~K}$ (see Figure 11(b)). However, Trojans have been estimated to have experienced temperatures as high as $180 \mathrm{~K}$ (e.g., Guilbert-Lepourtre 2014). The non-specular data up to well above $180 \mathrm{~K}$ (see Figures 9(d), 10(d), and 11(c) for up to $170 \mathrm{~K}$ ) also showed slightly steeper slopes for the 3 -ice $+\mathrm{H}_{2} \mathrm{~S}$, so the present results also support trend (1) for Trojans.

Trends (2) and (3) describe the spectral changes upon heating. The non-specular geometry results showed increasing signal up to about $160 \mathrm{~K}$, stable signal up to $180 \mathrm{~K}$, and then a major decrease in signal while heating above $180 \mathrm{~K}$. Therefore, only the warmest estimates for Trojans would match trend (2). Similarly, decreases in slope compared to the $50 \mathrm{~K}$ data were only observed above $150 \mathrm{~K}$, so trend (3) was also only supported for warmest estimates of Trojan surface temperature histories.

Based on the above analysis, the laboratory examinations reported here support trend (1) of the sulfur hypothesis and may support trends (2) and (3). However, the lab results contained additional details. For example, the lab results showed prominent absorption bands at 410, 620, and $900 \mathrm{~nm}$ for the 3-ice $+\mathrm{H}_{2} \mathrm{~S}$, which have not been reported for any KBO or Trojan objects. These prominent bands would be visible on large KBOs, but only small KBOs show the color bimodality addressed by the present hypothesis (e.g., Brown et al. 2011). There are no published spectra of KBOs in the relevant size range and with sufficient spectral and radiometric resolution to resolve these bands. Second, the present results require Trojans to have had a "warm" history in order to support the hypothesis, but according to the present results, a "warm" history would also have destroyed the absorption bands. Therefore, the lack of observed absorption bands on the Trojans is consistent with the laboratory conditions where the hypothesis is supported, i.e., a warm thermal history.

A second detail had to do with changes to the physical structure of the sample at the conditions where the experiments supported trends (2) and (3). During heating from 140 to $200 \mathrm{~K}$ the unreacted ice mixtures became volatile and sublimed out of the sample. Care was taken to keep this process very slow, so as not to disrupt the refractory residue more than necessary (nor over-pressurize any sensitive components of the instrumentation), but the conditions where volatiles would have been lost on a Trojan object are unknown. It may have been gentle, or it may have involved jets like a comet. Disruptive loss of volatiles may alter the morphology of the surface and create geometries that affect the spectral slope. This uncertainty limits application of the experiments in the range where they showed the best match to the hypothesis.

A related limitation was that trends (2) and (3) were only supported by data collected after irradiation had been concluded. We did not test continued irradiation at high temperatures. In addition, the irradiation flux used here was many orders of magnitude higher than that at a real solar system body. This is a common challenge for laboratory 
Table 2

Changes in Spectral Slope and Apparent Reflectance During Key Phases of Each Experiment

\begin{tabular}{|c|c|c|c|c|c|c|c|c|c|}
\hline \multicolumn{2}{|c|}{ Gas Mixture Composition } & \multicolumn{2}{|c|}{ Thickness $(\mu \mathrm{m})$} & \multicolumn{3}{|c|}{ Change in Slope } & \multicolumn{3}{|c|}{ Change in Apparent Reflectance } \\
\hline Gases & Ratio & & $+/-$ & at $50 \mathrm{~K}$ & Heating to $120 \mathrm{~K}$ & at $120 \mathrm{~K}$ & at $50 \mathrm{~K}$ & Heating to $120 \mathrm{~K}$ & at $120 \mathrm{~K}$ \\
\hline $\mathrm{CH}_{3} \mathrm{OH}$ & pure & 2 & 1 & $\mathrm{R}$ & $\mathrm{R}$ & $\mathrm{n}^{\mathrm{a}}$ & $\mathrm{D}$ & $\mathrm{D}$ & $\mathrm{n}^{\mathrm{a}}$ \\
\hline Round 1: Specular & & & & & & & & & \\
\hline $\mathrm{H}_{2} \mathrm{O}: \mathrm{CH}_{3} \mathrm{OH}: \mathrm{NH}_{3}$ & $1: 2: 2$ & 2 & 1 & $\mathrm{R}$ & $\mathrm{r}$ & $\mathrm{r}$ & $\mathrm{D}$ & $\mathrm{d}$ & $\mathrm{d}$ \\
\hline $\mathrm{H}_{2} \mathrm{O}: \mathrm{CH}_{3} \mathrm{OH}: \mathrm{NH}_{3}: \mathrm{H}_{2} \mathrm{~S}$ & $1: 3: 3: 3$ & 2 & 1 & $\mathrm{r}$ & $\mathrm{n}$ & $\mathrm{n}$ & $\mathrm{D}$ & d & d \\
\hline $\begin{array}{l}\mathrm{H}_{2} \mathrm{O}: \mathrm{CH}_{3} \mathrm{OH}: \mathrm{NH}_{3}: \mathrm{H}_{2} \mathrm{~S} \\
\text { Round 2: Specular }\end{array}$ & $1: 3: 3: 3$ & 2 & 1 & $\mathrm{R}$ & $\mathrm{r}$ & $\mathrm{r}$ & $\mathrm{D}$ & d & $\mathrm{D}$ \\
\hline$\overline{\mathrm{H}_{2} \mathrm{O}: \mathrm{CH}_{3} \mathrm{OH}: \mathrm{N}_{3}}$ & $1: 2: 2$ & 40 & 10 & $\mathrm{n}$ & $\mathrm{n}$ & $\mathrm{n}$ & $\mathrm{D}$ & $\mathrm{d}$ & $\mathrm{n}$ \\
\hline $\begin{array}{l}\mathrm{H}_{2} \mathrm{O}: \mathrm{CH}_{3} \mathrm{OH}: \mathrm{NH}_{3}: \mathrm{H}_{2} \mathrm{~S} \\
\text { Round 3: Non-specular }\end{array}$ & $1: 3: 3: 3$ & 200 & 80 & $\mathbf{R}$ & $\mathrm{r}$ & $\mathrm{b}$ & D & $\mathrm{n}$ & $\mathrm{n}$ \\
\hline $\mathrm{H}_{2} \mathrm{O}: \mathrm{CH}_{3} \mathrm{OH}: \mathrm{NH}_{3}$ & $1: 2: 2$ & 8 & 3 & $\mathrm{R}$ & $\mathrm{r}$ & $b^{b}$ & $I^{c}$ & $\mathrm{~d}$ & $d^{b}$ \\
\hline $\mathrm{H}_{2} \mathrm{O}: \mathrm{CH}_{3} \mathrm{OH}: \mathrm{NH}_{3}: \mathrm{H}_{2} \mathrm{~S}$ & $1: 3: 3: 3$ & 60 & 20 & $\mathbf{R}$ & $\mathrm{n}$ & $n^{\mathrm{b}}$ & $\mathbf{I}^{\mathrm{c}}$ & $\mathrm{d}$ & $n^{\mathrm{b}}$ \\
\hline
\end{tabular}

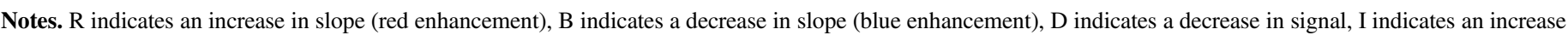

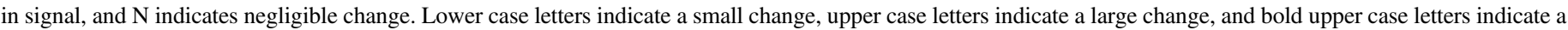
very large change. See the text for changes during heating after irradiation had concluded.

a $150 \mathrm{~K}$ was used instead of $120 \mathrm{~K}$ for the methanol.

b There were negligible changes during the $142 \mathrm{~K}$ irradiation phase of the Round 3: Non-specular experiments.

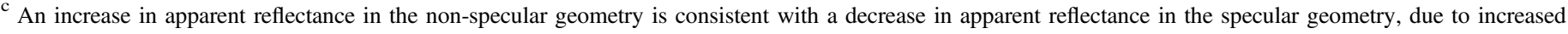
scattering.

irradiation experiments simulating slow processes. Experiments varying irradiation flux and irradiating at higher temperatures may help to address these sources of uncertainty in application.

An additional limitation of applying the present experiments to solar system objects is that only thermal and radiation ( $10 \mathrm{keV}$ electron) processing were simulated by the laboratory experiments. This neglected the effects of impact gardening and other resurfacing processes. Impact gardening should completely devolatilize portions of the surface and lead to objects with surface colors intermediate between the LR and R Trojan spectral groups, yet few objects in the gap have been observed (Wong et al. 2014). This is not well understood and requires further examination.

Finally, a possible detection of a silicate absorption feature at some Trojans has been reported (Emery et al. 2006), although it was not confirmed by a separate investigation (Yang \& Jewitt 2011). Silicates containing iron typically exhibit "red" spectral slopes in the visible and near-infrared upon space weathering (e.g., Bennett et al. 2013). If silicates are contributing to the spectra of Trojan asteroids, then they are likely involved in defining the slope of these spectra. Material similar to C-type asteroids may also be included in the non-icy materials expected on the surfaces of Trojans, and could further darken Trojans and flatten the average spectral slope.

This first laboratory investigation into ice mixtures with and without sulfur provides new data to investigate a possible link between KBOs, Trojans, and solar system reorganization. The present work is broadly consistent with the hypothesized history of these bodies and new information-like the potential existence of visible absorptions due to Sulfur-containing species in ices-remains to be tested by comparison to telescopic observation. Further examination of mixed ices under additional experimental sequences, with varying ratios of the deposited ices, inclusion of likely materials not considered here, and additional astronomical observations may all be necessary to understand this complex scenario and vindicate or refute the sulfur hypothesis.

\subsection{Application to Other Bodies}

While the main purpose of this work was to test the above hypothesis explaining the color bimodality of Trojans and small KBOs, the results from spectroscopy of the complex ice mixtures may also be relevant to other solar system bodies, such as Io, Ceres, comets, Iapetus, and centaurs. Taking the case of Io, an apparent absorption has been reported by Geissler et al. (1999) at about $900 \mathrm{~nm}$ in some of the dark and the white regions on Io. Since Io is in a strong irradiation environment with abundant sulfur, this potential absorption may be related to the one observed in the present work. Water is unlikely to be abundant on Io, so the clathrate origin of the $900 \mathrm{~nm}$ feature proposed above would not be likely on Io and another source would need to be identified. However, several of the sources proposed by Geissler et al. included elements not available here; the present work suggests that the source of the $900 \mathrm{~nm}$ absorption could be due to a compound containing only S, N, $\mathrm{C}, \mathrm{O}$, and/or $\mathrm{H}$.

\section{Conclusions}

We successfully simulated the evolution in spectral properties of mixtures of water $\left(\mathrm{H}_{2} \mathrm{O}\right)$, methanol $\left(\mathrm{CH}_{3} \mathrm{OH}\right)$, and ammonia $\left(\mathrm{NH}_{3}\right)$-with and without hydrogen sulfide $\left(\mathrm{H}_{2} \mathrm{~S}\right)$ - over a temperature and irradiation history similar to that of a Trojan asteroid that had formed an irradiation crust at 20 au before being relocated to $5 \mathrm{au}$. These data largely support the hypothesis that the presence or absence of sulfur in the irradiated crust of Trojans and KBOs is the key difference between spectral groups of these small bodies in the middle and outer solar system (Wong \& Brown 2016). However, many limitations and uncertainties remain to be addressed before the hypothesis would be proven true. The first condition on the hypothesis - that S-bearing ices are redder following radiation -was supported by substantial reddening of the VNIR slope for irradiated ice mixtures containing $\mathrm{H}_{2} \mathrm{~S}$, and reddening of a lesser degree for similar irradiated ice mixtures without $\mathrm{H}_{2} \mathrm{~S}$. 
Darkening and decreased reddening upon heating are the other key predictions on the hypothesis. The present results only supported these conditions of the hypothesis in the experimentally complex region from 142 to $200 \mathrm{~K}$, which included both the conclusion of irradiation and the sublimation of unreacted ices. These temperatures are on the higher range of the predicted temperature histories of Trojans (maximum $180 \mathrm{~K}$ ). Prominent absorptions in the visible were also found for S-containing ices at KBO-like temperatures near $600 \mathrm{~nm}$, $900 \mathrm{~nm}$, and possibly $410 \mathrm{~nm}$. No astronomical data at sufficient spectral and radiometric resolution of the smallest KBOs yet exist to examine this laboratory result against astronomical data.

Future work might include adding nonvolatile, neutral (i.e., C-type asteroid material), or red (i.e., space-weathered silicates) materials in experiments to consider their effects on the products of chemical reactions occurring in response to irradiation. Therefore, while the present work largely supports the hypothesis of large-scale planetary migration to explain different populations of KBOs and Trojans of similar origin, significant additional experiment, theory, and astronomical observations are needed to fully test the hypothesis.

This work has been supported by the W. M. Keck Institute for Space Studies (KISS). This work has been conducted at the Jet Propulsion Laboratory, Caltech, under a contract with the National Aeronautics and Space Administration (NASA) and at the Caltech Division of Geological and Planetary Sciences.

\section{ORCID iDs}

Michael J. Poston (1D https://orcid.org/0000-0001-5113-1017 Ahmed Mahjoub (1D https://orcid.org/0000-0003-1229-5208 Bethany L. Ehlmann (i) https://orcid.org/0000-00022745-3240

Michael E. Brown (1D https://orcid.org/0000-0002-8255-0545

Kevin P. Hand (10 https://orcid.org/0000-0002-3225-9426

Ian Wong (1) https://orcid.org/0000-0001-9665-8429

\section{References}

Andronico, G., Baratta, G. A., Spinella, F., \& Strazzulla, G. 1987, A\&A, 184,333

Bennett, C. J., Pirim, C., \& Orlando, T. M. 2013, ChRv, 113, 9086

Brown, M. E., Schaller, E. L., \& Fraser, W. C. 2011, ApJL, 739, L60

Brunetto, R., Barucci, M. A., Dotto, E., \& Strazzulla, G. 2006, ApJ, 644, 646

Carlson, R. W., Calvin, W. M., Dalton, J. B., et al. 2009, in Europa, ed. R. T. Pappalardo, W. B. Mckinnon, \& K. K. Khurana (Tucson, AZ: Univ. Arizona Press), 283

Carlson, R. W., Kargel, J. S., Doute, S., Soderblom, L. A., \& Dalton, J. B. 2007, in Io After Galileo, ed. R. M. C. Lopes \& J. R. Spencer (Chichester: Praxis Publishing Ltd.), https://doi.org/10.1007/978-3-540-48841-5

Collings, M. P., Anderson, M. A., Chen, R., et al. 2004, MNRAS, 354, 1133

DeSanctis, M. C., Ammannito, E., Raponi, A., et al. 2015, Natur, 528, 241

Eckert, B., \& Steudel, R. 2003, in Elemental Sulfure and Sulfur-Rich Compounds II, ed. R. Steudel (Berlin: Springer), 31

Emery, J. P., Burr, D. M., \& Cruikshank, D. P. 2011, AJ, 141, 25

Emery, J. P., Cruikshank, D. P., \& Van Cleve, J. 2006, Icar, 182, 496

Geissler, P. E., McEwen, A. S., Keszthelyi, L., et al. 1999, Icar, 140, 265

Guilbert-Lepourtre, A. 2014, Icar, 231, 232

Hand, K. P. 2007, PhD thesis, Stanford Univ.

Hand, K. P., \& Carlson, R. W. 2011, Icar, 215, 226

Hand, K. P., \& Carlson, R. W. 2012, JGR, 117, E03008

Hudson, R. L., Palumbo, M. E., Strazzulla, G., et al. 2008, in The Solar System Beyond Neptune, ed. M. A. Barucci et al. (Tucson, AZ: Univ. Arizona Press), 507

Johnson, P. B., \& Christy, R. W. 1972, PhRvB, 6, 4370

Lebofsky, L. A., \& Fegley, M. B., Jr. 1976, Icar, 28, 379

Levison, H. F., Bottke, W. F., Gounelle, M., et al. 2009, Natur, 460, 364

Mahjoub, A., Poston, M. J., Hand, K. P., et al. 2016, ApJ, 820, 141

Mahjoub, A., Poston, M. J., Hand, K. P., et al. 2017, ApJ, 846, 148

McCord, T. B., Castillo-Rogez, J., \& Rivkin, A. 2011, SSRv, 163, 63

McKinnon, W. B. 2008, Asteroids Comets Meteors (LPI Contrib.) Paper 8389

Meyer, B. 1976, ChRv, 76, 3

Morbidelli, A., Levison, H. F., Tsiganis, K., \& Gomes, R. 2005, Natur, 435, 462

Nesvorný, D., Vokrouhlický, D., \& Morbidelli, A. 2013, ApJ, 768, 45

Pieters, C. M., \& Noble, S. K. 2016, JGR, 121, 1865

Poston, M. J., Carlson, R. W., \& Hand, K. P. 2017, JGR, 122, 2644

Roig, F., Ribeiro, A. O., \& Gil-Hutton, R. 2008, A\&A, 483, 911

Thompson, W. R., Murray, B. G. J. P. T., Khare, B. N., \& Sagan, C. 1987, JGR, 92, 14933

Wong, I., \& Brown, M. E. 2016, AJ, 152, 90

Wong, I., \& Brown, M. E. 2017, AJ, 153, 145

Wong, I., Brown, M. E., \& Emery, J. P. 2014, AJ, 148, 112

Yang, B., \& Jewitt, D. 2011, AJ, 141, 95 